\title{
The Paternal Mental Health, Difficulties for Fathers with Children in Early Infancy, and Their Educational Support Needs
}

\author{
Kimiko Higashio',2, Ayako Sasaki ${ }^{3}$ \\ ${ }^{1}$ Graduate School of Nursing, Osaka Medical and Pharmaceutical University, Osaka, Japan \\ ${ }^{2}$ Faculty of Nursing, School of Nursing, Takarazuka University, Osaka, Japan \\ ${ }^{3}$ Faculty of Nursing, Osaka Medical and Pharmaceutical University, Osaka, Japan \\ Email: k-higashio@takara-univ.ac.jp
}

How to cite this paper: Higashio, $K$. and Sasaki, A. (2021) The Paternal Mental Health, Difficulties for Fathers with Children in Early Infancy, and Their Educational Support Needs. Health, 13, 789-811. https://doi.org/10.4236/health.2021.138061

Received: July 10, 2021

Accepted: August 2, 2021

Published: August 5, 2021

Copyright ( 2021 by author(s) and Scientific Research Publishing Inc. This work is licensed under the Creative Commons Attribution International License (CC BY 4.0).

http://creativecommons.org/licenses/by/4.0/ (c) (i) Open Access

\begin{abstract}
Purpose: The purpose is to identify what difficulties fathers have with the roles of fatherhood during pregnancy and early infancy, and to make apparent what their educational support needs are. Methods: This study follows a qualitative descriptive design. The subjects were new first-time fathers of Japanese nationality. Subjects participated in semi-structured interviews administered according to interview guidelines. Results: There were 15 subjects in total. The average age of the subjects' children was 5.5 months. Fathers' difficulties with their new roles were abstracted in to five main categories: $<$ Difficulties during early engagement with child $>,<$ Difficulties related to child crying $>,<$ Difficulties arising after getting through the early engagement stage $>$, $<$ Difficulties related to support of partner $>$, and $<$ Mental burdens associated with childrearing $>$. Additionally, the types of support fathers requested to help maintain their mental health after the birth of their infants were abstracted into three main categories: <Support with childcare knowledge/acquiring skills $>$, <Support in building a positive marital relationship $>$, and $<$ Support in maintaining own mental health $>$. Conclusions: 1 ) The stress experienced by fathers from the birth of their child through early infancy, originating in two elements of fatherhood (engagement with the child and spousal support), placed a great mental burden upon them in a multi-layered structure; 2) The support that needs fathers expressed to maintain their mental health pre- and postpartum is based on the difficulties they experienced with paternal roles; 3) There is a disparity between the needs of fathers expressed through the results of this study and current paternal support practices in Japan. The supplementation and revision of existing paternal support practices is an issue that merits further consideration. Furthermore, the existing group interview
\end{abstract}


method suffers from low attendance, and attending has become increasingly difficult due to the COVID-19 pandemic spreading throughout Japan. It is necessary to consider alternative methods of educational support moving forward.

\section{Keywords}

Paternity, Fatherhood, Paternity Support, Paternal Mental Health, Difficulties with Fatherhood

\section{Introduction}

\subsection{Academic Background of Study}

After falling below 1 million births in 2016, the birthrate in Japan continues to decline, reaching only 865,234 in 2019 [1]. It has been claimed that the declining marriage and increase in average age of marriage are causes of the declining birth rate [2]. Furthermore, the increase in average age of childbirth and the shrinking population are influencing economic contraction and labor shortages. In the face of these issues, combating the declining birth rate is of utmost importance in Japan.

Since the so-called "1.57 Shock" of 1990, the Japanese government has employed a number of policies to combat the low birth rate. In 1999, in response to the declining birth rate, aging population, and economic changes, the Basic Law for a Gender-Equal Society was implemented in an effort to create a society in which citizens would have better opportunity to demonstrate their own unique talents without regard for gender. In addition, the Ministry of Health, Labour, and Welfare started the "Ikumen Project," a policy designed to encourage fathers to be more involved with childcare and help them get paternity leave, in 2010 . The Outline of Measures for a Low-Birth-Rate Society was formed in 2015. In the following year, labor reforms and childcare reforms aimed at eliminating childcare placement waiting lists were planned based on the Plan for Dynamic Engagement of All Citizens [3], as well as the Fourth Basic Plan for Gender Equality, which is designed to encourage equal opportunities for men and women in the workplace, society, and the home. In 2017, the New Economic Policy Package was introduced to reduce the financial burden on the childrearing generation [4].

Despite these numerous reforms set forth by the Japanese government, the situation remains bleak, with financial instability among parents, long working hours, and a shortage of childcare facilities leading to a large number of mothers shouldering the burden of childcare themselves [5]. While the percentage of men taking paternity leave reached its highest ever in fiscal 2019 at 7.48\%, this was only a small increase [6]. In 2020, the Fifth Basic Plan for Gender Equality was decided upon by the Cabinet with the goal of attaining a vital and sustainable 
society [7]. The effects of these efforts will be a challenge moving forward.

Parents currently raising children face a wide variety of serious issues. Changes to the childrearing environment place a significant burden on parents. Parents have little experience with infants due to the declining birth rate, the trend toward nuclear families has diminished the quality of care in households, and urbanization has reduced the availability of community support for childrearing. Parents are raising their children while shouldering the anxieties and concerns they have about how to do so, which negatively affects their mental health.

Some critical issues concerning maternal mental health are depression and child abuse brought on by the anxiety and stress of raising children. These have developed into major societal problems. The Japanese government is pushing the Healthy Parents and Children 21 (Second Phase) plan focused on the key issues of supporting parents in consideration of the difficulty of raising children and preventing child abuse from pregnancy through early childhood. In concrete terms, 2017 reforms to Maternal and Child Health Law aim to open "Comprehensive Support Centers for Families with Children" nationwide in order to provide seamless support to mothers from pregnancy and childbirth through the childrearing period.

In addition to the issues mentioned above, mental health issues affecting fathers have become apparent recent years. More fathers than mothers lack confidence in their child care skills, with $23.8 \%$ saying so from 3 - 4 months, increasing to $27.9 \%$ from $9-10$ months [8]. It has been reported that anxiety is at its strongest with the first child compared to families with multiple children [9], that the anxieties which form during pregnancy continue to be present at six months after birth [10], and that child care anxieties at one month after birth are associated with depressive states [11]. Additionally, about $10 \%$ of fathers are in a depressive state before or after birth. The period from three months to six months postpartum has particularly high rates, reported at $13.2 \%$ [12] and $25.6 \%$ [13]. Paternal depression has also been reported to be related to the corporal punishment of children [14].

In summary, during the first year of life, parental roles are filled not only by the mother but by the father as well, with a great effect on their mental health. After the birth, first-time parents, regardless of gender, must face these new roles as parents in addition to those which they already had. During pregnancy, mothers experience the growth of their child as a physical sensation, building an awareness of their role as a parent. There also exist many opportunities for mothers to obtain information about raising children, which enables them to prepare for the transition to being a parent. However, fathers are saddled with their paternal role only after birth, and if they are unable to cope, it can lead to a deterioration of mental health.

The Fifth Basic Plan for Gender Equality was presented as a concrete measure to increase men's involvement in childrearing and to provide indirect support for new fathers from the pregnancy onward. Accordingly, nurses are expected to 
fill the vital role of providing support for men from the pregnancy onward so they can transition to life as a parent without difficulty [15].

Previous research has classified educational support encouraging paternal roles based on the goal: encouraging self-awareness of the father, encouraging understanding and support for the mother following the birth, encouraging childcare activity in the father, and encouraging engagement with the child. It has been shown that it is most effective to begin encouraging childcare activity during the pregnancy, and research suggests that there is a need to simultaneously provide emotional support and continuous measures to help fathers cope with the anxieties of childcare. However, the difficulties fathers have with childrearing upon which this support would be based are ambiguous, indicating the importance of understanding these needs and of support programs designed to deal with them [16]. Furthermore, while the need for prevention and support efforts to combat the poor state of paternal mental health has been raised [17], it has not been sufficiently verified.

\subsection{Purpose of Study}

The purpose of this study is to attempt to identify what difficulties fathers have with the roles of fatherhood during pregnancy and early infancy, as well as what their educational support needs are.

\subsection{Terminology}

1) Father: Men who live with a partner, regardless of marital status, and said partner is pregnant or a child of less than one year of age is present.

2) Mother: Women who live with a partner and are pregnant with said partner's child or said partner's child is present.

3) Paternal roles: Modern paternal roles have been classified into the following responsibilities: providing financial support, helping with housework, taking care of the child [18], socializing the child, and being a caregiver [19]. Okada, et al. define the paternal roles during infancy as being prepared to protect the family; providing support to lessen the mother's burden, taking care of the child, considering child care styles, and bearing the economic burden during the transition to life with children [20]. Based on these, in this study, we define paternal roles as the responsibilities made up of these four factors: self-awareness as a father, understanding and support for mother, childcare activity, and infant engagement.

4) Paternal mental health: In this study, when we refer to maintaining paternal mental health, we speak with regard to the ability of fathers to cope with the difficulties associated with the roles of fatherhood and to execute those roles, based on WHO definitions of mental health.

5) Difficulties associated with paternal roles: The difficulties fathers face in relation to the child and the mother. Concerning the child, these include feelings of confusion, anxiety, distress, and frustration due to a lack of childcare know- 
ledge and skills. Concerning the mother, these include confusion, anxiety, distress, and frustration that arise from parental expectations and the disparity between spousal expectations.

\section{Methods}

1) Study Design

It is a qualitative descriptive study.

2) Subjects

15 Japanese fathers raising their first child, who were at least 20 years of age and took less than one month of paternity leave, and for whose partner it was their first birth (single birth with no serious complications).

3) Method of Recruitment

We enlisted the help of a clinic in Osaka Prefecture in recruiting subjects.

a) Staff suggested the program to mothers at the time of discharge. Flyers were also placed in the maternity ward waiting room.

b) Mothers who showed interest in the program showed the flyers to the father. If the father consented, he would be directed to apply with the ward staff.

c) Researchers explained the study to the mother using instructional materials.

d) The mother was requested to give the instructional materials to the father. Contact information was verified.

e) Researchers contacted the father to confirm the time of the interview and adjust the desired time of the session.

4) Data Collection Period

It is between August 2020 - March 2021 (Interviews ran from December 2020 - March 2021).

5) Interview Method

i) The subject arrived at the clinic at the adjusted time. Based on the instructional materials, researchers explained the following aspects of the study: goal, method, voluntariness, protection of privacy, handling of personal information, guarantee of withdrawal, and publishing of study results. Consent was obtained by the signing of consent forms.

ii) The subject was interviewed privately in a private room so they could speak freely. The subject's consent to record the interview with a digital voice recorder was obtained. It was explained that the interview would take approximately 50 minutes.

iii) A semi-structured interview was conducted in accordance with interview guidelines.

At the start of the interview, researchers confirmed the following details to ascertain the subject's attributes: age of the child; age and occupational status of the mother; age of the father; time of going to work; time of returning from work; system of workdays/off days; daily housework on workdays as well as off days, hours taking care of child, and what is done while taking care of child.

The interview included the following topics: 
a) Issues having to do with the relationship with the child in the time since the mother was pregnant until six months after the child was born.

b) Issues having to do with the relationship with the mother in the time since she became pregnant until six months after the child was born.

c) Desired education around the time of birth for the sake of maintaining mental health.

6) Method of Analysis

Analysis was conducted based on Berelson's content analysis method [21]. This method of analysis is considered "a research technique for the objective, systematic, and quantitative description of the manifest content of communication." [21] We chose this method for this study because analyzing the content of linguistic communication is the approach most suited to capturing the varied and difficult-to-quantify narrative of the fathers' experiences.

Data was extracted from transcripts of the interview recordings. Each line of thought a subject spoke of about the four factors of paternal responsibility (self-awareness as a father, understanding and support for mother, childcare activity, and infant engagement) was made a recording unit. If one line of thought contained multiple difficulties originating from the four factors of paternal responsibility, it was split into two recording units. In order to prevent a change in the meaning of the recording units, they were expressed as one sentence. Recording units were gathered, and their contents classified according to their similarity. These contents were then abstracted and coded. The semantic content of the codes was compared, and they were categorized according to their similarity. These categories were further abstracted into a three-level hierarchy of categories.

Additionally, each line of thought concerning necessary support before and after pregnancy was made a recording unit. In the same way as it was for difficulties originating in the four factors of paternal responsibility, the content was abstracted and classified based on similarity.

The entire analysis process was supervised by an advisor.

7) Ethical Considerations

Subjects were told in writing as well as verbally the intent, objectives, and method of the study. It was communicated to the subjects that participation was voluntary, that subjects would not be penalized for early withdrawal, that their privacy would be preserved upon publishing, with all data remaining strictly anonymous, and that this data would be managed with utmost care. Subjects' consent was obtained in writing. In addition, care was taken to consider the subject's physical and mental condition and a support system was in place in case any issues were to arise. This study was conducted with the approval of the Osaka Medical and Pharmaceutical University ethics committee and the ethics boards of all cooperating institutions.

\section{Results}

1) Subject Characteristics (Table 1) 
Table 1. Outline of subject characteristics.

\begin{tabular}{|c|c|c|c|c|c|c|c|c|c|c|c|c|c|c|c|c|}
\hline \multirow{3}{*}{ Subject } & \multirow{3}{*}{$\begin{array}{l}\text { Age } \\
\text { group }\end{array}$} & \multirow{3}{*}{$\begin{array}{l}\text { Leaves } \\
\text { for work }\end{array}$} & \multirow{3}{*}{$\begin{array}{l}\text { Returns } \\
\text { home }\end{array}$} & \multirow{3}{*}{$\begin{array}{l}\text { Day off } \\
\text { system }\end{array}$} & \multicolumn{4}{|c|}{ Housework } & \multicolumn{4}{|c|}{ Child care } & \multirow{3}{*}{$\begin{array}{l}\text { Mother's } \\
\text { age } \\
\text { group }\end{array}$} & \multirow{3}{*}{$\begin{array}{c}\text { Mother's } \\
\text { employment } \\
\text { status }\end{array}$} & \multirow{3}{*}{$\begin{array}{l}\text { Age of } \\
\text { child }\end{array}$} & \multirow{3}{*}{$\begin{array}{c}\text { Interview } \\
\text { duration } \\
(\min )\end{array}$} \\
\hline & & & & & \multicolumn{2}{|c|}{ Work days } & \multicolumn{2}{|c|}{ Days off } & \multicolumn{2}{|c|}{ Work days } & \multicolumn{2}{|r|}{ Days off } & & & & \\
\hline & & & & & Hours & Content & Hours & Content & Hours & Content & Hours & Content & & & & \\
\hline A & $30 \mathrm{~s}$ & 8:00 & $21: 00$ & $\begin{array}{l}\text { Sat/Sun/ } \\
\text { Holidays }\end{array}$ & 1 & $\begin{array}{l}\text { cleaning, } \\
\text { taking out } \\
\text { trash, } \\
\text { shopping }\end{array}$ & 1 & $\begin{array}{l}\text { cooking, } \\
\text { shopping }\end{array}$ & 2 & $\begin{array}{l}\text { changing } \\
\text { diaper, } \\
\text { giving bath, } \\
\text { comforting, } \\
\text { putting to } \\
\text { sleep, } \\
\text { playing }\end{array}$ & 8 & $\begin{array}{l}\text { changing } \\
\text { diaper, } \\
\text { giving bath, } \\
\text { comforting, } \\
\text { playing }\end{array}$ & $20 \mathrm{~s}$ & Unemployed & 8 & 51 \\
\hline B & $30 \mathrm{~s}$ & $6: 00$ & $21: 00$ & $\begin{array}{l}2 \text { days } \\
\text { off/week }\end{array}$ & 0.5 & $\begin{array}{l}\text { taking out } \\
\text { trash }\end{array}$ & 1 & $\begin{array}{l}\text { taking } \\
\text { out } \\
\text { trash }\end{array}$ & 0 & none & 5 & $\begin{array}{l}\text { changing } \\
\text { diaper, } \\
\text { giving bath, } \\
\text { comforting, } \\
\text { playing }\end{array}$ & $30 \mathrm{~s}$ & Unemployed & 8 & 61 \\
\hline $\mathrm{C}$ & $20 \mathrm{~s}$ & $8: 00$ & $18: 00$ & $\begin{array}{l}\text { Sat/Sun/ } \\
\text { Holidays }\end{array}$ & 1 & $\begin{array}{l}\text { laundry, } \\
\text { cleaning, } \\
\text { shopping }\end{array}$ & 2 & $\begin{array}{l}\text { cooking, } \\
\text { cleaning, } \\
\text { shopping }\end{array}$ & 1.5 & $\begin{array}{l}\text { changing } \\
\text { diaper, } \\
\text { comforting, } \\
\text { putting to } \\
\text { sleep, } \\
\text { playing }\end{array}$ & 3 & $\begin{array}{l}\text { changing } \\
\text { diaper, } \\
\text { giving bath, } \\
\text { comforting, } \\
\text { putting to } \\
\text { sleep, } \\
\text { playing }\end{array}$ & $20 \mathrm{~s}$ & $\begin{array}{l}\text { On } \\
\text { maternity } \\
\text { leave }\end{array}$ & 5 & 53 \\
\hline $\mathrm{D}$ & $20 \mathrm{~s}$ & $8: 00$ & 19:00 & $\begin{array}{l}\text { Sat/Sun/ } \\
\text { Holidays }\end{array}$ & 1 & $\begin{array}{l}\text { laundry, } \\
\text { cleaning, } \\
\text { taking out } \\
\text { trash }\end{array}$ & 1 & $\begin{array}{l}\text { cooking, } \\
\text { laundry, } \\
\text { taking } \\
\text { out } \\
\text { trash }\end{array}$ & 4 & $\begin{array}{l}\text { feeding, } \\
\text { changing } \\
\text { diaper, } \\
\text { giving bath, } \\
\text { comforting, } \\
\text { putting to } \\
\text { sleep, } \\
\text { playing }\end{array}$ & , & $\begin{array}{l}\text { feeding, } \\
\text { changing } \\
\text { diaper, } \\
\text { giving bath, } \\
\text { comforting, } \\
\text { putting to } \\
\text { sleep, } \\
\text { playing }\end{array}$ & $20 \mathrm{~s}$ & $\begin{array}{l}\text { On } \\
\text { maternity } \\
\text { leave }\end{array}$ & 4 & 61 \\
\hline E & $30 \mathrm{~s}$ & $8: 00$ & 19:00 & $\begin{array}{c}2 \text { days/ } \\
\text { week }\end{array}$ & 1 & $\begin{array}{l}\text { laundry, } \\
\text { cleaning, } \\
\text { taking } \\
\text { out trash }\end{array}$ & 1 & $\begin{array}{l}\text { cooking, } \\
\text { laundry, } \\
\text { cleaning, } \\
\text { taking } \\
\text { out } \\
\text { trash, } \\
\text { shopping }\end{array}$ & 1 & $\begin{array}{l}\text { feeding, } \\
\text { changing } \\
\text { diaper, } \\
\text { giving bath, } \\
\text { comforting, } \\
\text { putting } \\
\text { to sleep }\end{array}$ & , & $\begin{array}{l}\text { feeding, } \\
\text { changing } \\
\text { diaper, } \\
\text { giving bath, } \\
\text { comforting, } \\
\text { putting } \\
\text { to sleep }\end{array}$ & $30 \mathrm{~s}$ & $\begin{array}{l}\text { On } \\
\text { maternity } \\
\text { leave }\end{array}$ & 2 & 45 \\
\hline $\mathrm{F}$ & $30 \mathrm{~s}$ & 8:00 & $\begin{array}{c}17: 00- \\
23: 00\end{array}$ & $\begin{array}{c}1-2 \\
\text { days/ } \\
\text { week }\end{array}$ & 1 & $\begin{array}{l}\text { laundry, } \\
\text { taking out } \\
\text { trash, } \\
\text { shopping }\end{array}$ & 1 & $\begin{array}{l}\text { laundry, } \\
\text { taking } \\
\text { out } \\
\text { trash, } \\
\text { shopping }\end{array}$ & 3 & $\begin{array}{l}\text { feeding, } \\
\text { changing } \\
\text { diaper, } \\
\text { giving bath, } \\
\text { comforting, } \\
\text { putting to } \\
\text { sleep, } \\
\text { playing }\end{array}$ & , & $\begin{array}{l}\text { feeding, } \\
\text { changing } \\
\text { diaper, } \\
\text { giving bath, } \\
\text { comforting, } \\
\text { putting to } \\
\text { sleep, } \\
\text { playing }\end{array}$ & $30 \mathrm{~s}$ & Full-time & 11 & 52 \\
\hline G & $30 \mathrm{~s}$ & $7: 00$ & $18: 00$ & $\begin{array}{l}1 \text { day/ } \\
\text { week }\end{array}$ & 1 & $\begin{array}{l}\text { laundry, } \\
\text { taking out } \\
\text { trash, } \\
\text { doing } \\
\text { dishes }\end{array}$ & 1 & $\begin{array}{l}\text { laundry, } \\
\text { taking } \\
\text { out } \\
\text { trash, } \\
\text { doing } \\
\text { dishes, } \\
\text { shopping }\end{array}$ & 2 & $\begin{array}{l}\text { feeding, } \\
\text { changing } \\
\text { diaper, } \\
\text { giving bath, } \\
\text { comforting, } \\
\text { putting } \\
\text { to sleep }\end{array}$ & , & $\begin{array}{l}\text { feeding, } \\
\text { changing } \\
\text { diaper, } \\
\text { giving bath, } \\
\text { comforting, } \\
\text { putting } \\
\text { to sleep }\end{array}$ & $30 \mathrm{~s}$ & Unemployed & 5 & 65 \\
\hline
\end{tabular}




\section{Continued}

\begin{tabular}{|c|c|c|c|c|c|c|c|c|c|c|c|c|c|c|c|c|}
\hline $\mathrm{H}$ & $20 \mathrm{~s}$ & $6: 00$ & $21: 00$ & $\begin{array}{l}1 \text { day/ } \\
\text { week }\end{array}$ & 1 & $\begin{array}{l}\text { cleaning } \\
\text { bathtub, } \\
\text { taking out } \\
\text { trash }\end{array}$ & 1 & $\begin{array}{l}\text { laundry, } \\
\text { cleaning, } \\
\text { taking } \\
\text { out } \\
\text { trash }\end{array}$ & 1 & $\begin{array}{l}\text { changing } \\
\text { diaper, } \\
\text { giving bath, } \\
\text { comforting, } \\
\text { putting } \\
\text { to sleep }\end{array}$ & 6 & $\begin{array}{l}\text { changing } \\
\text { diaper, } \\
\text { giving bath, } \\
\text { comforting, } \\
\text { putting to } \\
\text { sleep, } \\
\text { playing }\end{array}$ & $20 \mathrm{~s}$ & $\begin{array}{l}\text { On } \\
\text { maternity } \\
\text { leave }\end{array}$ & 4 & 49 \\
\hline I & $30 \mathrm{~s}$ & $7: 00$ & $\begin{array}{l}\text { 19:00 } \\
\text { (shift) }\end{array}$ & $\begin{array}{l}\text { Sat/Sun/ } \\
\text { Holidays }\end{array}$ & 1 & $\begin{array}{l}\text { taking out } \\
\text { trash }\end{array}$ & 2 & $\begin{array}{l}\text { taking } \\
\text { out } \\
\text { trash, } \\
\text { shopping }\end{array}$ & 2 & $\begin{array}{l}\text { feeding, } \\
\text { changing } \\
\text { diaper, } \\
\text { giving bath, } \\
\text { comforting, } \\
\text { putting to } \\
\text { sleep, } \\
\text { playing }\end{array}$ & 3 & $\begin{array}{l}\text { feeding, } \\
\text { changing } \\
\text { diaper, } \\
\text { giving bath, } \\
\text { comforting, } \\
\text { putting to } \\
\text { sleep, } \\
\text { playing }\end{array}$ & $30 \mathrm{~s}$ & $\begin{array}{l}\text { On } \\
\text { maternity } \\
\text { leave }\end{array}$ & 6 & 74 \\
\hline $\mathrm{J}$ & $30 s$ & $7: 00$ & 19:00 & $\begin{array}{l}1 \text { day/ } \\
\text { week }\end{array}$ & 4 & $\begin{array}{l}\text { laundry, } \\
\text { taking out } \\
\text { trash, } \\
\text { doing } \\
\text { dishes }\end{array}$ & 6 & $\begin{array}{l}\text { cooking, } \\
\text { laundry, } \\
\text { shopping }\end{array}$ & 2 & $\begin{array}{l}\text { feeding, } \\
\text { changing } \\
\text { diaper, } \\
\text { giving bath, } \\
\text { comforting, } \\
\text { putting to } \\
\text { sleep, } \\
\text { playing }\end{array}$ & 6 & $\begin{array}{l}\text { feeding, } \\
\text { changing } \\
\text { diaper, } \\
\text { giving bath, } \\
\text { comforting, } \\
\text { putting to } \\
\text { sleep, } \\
\text { playing }\end{array}$ & $30 \mathrm{~s}$ & $\begin{array}{l}\text { On } \\
\text { maternity } \\
\text { leave }\end{array}$ & 5 & 47 \\
\hline $\mathrm{K}$ & $30 \mathrm{~s}$ & $5: 00$ & $18: 00$ & $\begin{array}{l}1 \text { day/ } \\
\text { week }\end{array}$ & 1 & cleaning & 2 & $\begin{array}{l}\text { laundry, } \\
\text { cleaning, } \\
\text { taking } \\
\text { out } \\
\text { trash }\end{array}$ & 2 & $\begin{array}{l}\text { feeding, } \\
\text { changing } \\
\text { diaper, } \\
\text { giving bath, } \\
\text { comforting, } \\
\text { putting to } \\
\text { sleep, } \\
\text { playing }\end{array}$ & 5 & $\begin{array}{l}\text { feeding, } \\
\text { changing } \\
\text { diaper, } \\
\text { giving bath, } \\
\text { comforting, } \\
\text { putting to } \\
\text { sleep, } \\
\text { playing }\end{array}$ & $30 \mathrm{~s}$ & Unemployed & 5 & 56 \\
\hline $\mathrm{L}$ & $30 \mathrm{~s}$ & $9: 00$ & $22: 00$ & $\begin{array}{l}2 \text { days/ } \\
\text { week }\end{array}$ & 0 & none & 1 & $\begin{array}{l}\text { cleaning, } \\
\text { taking } \\
\text { out } \\
\text { trash, } \\
\text { shopping }\end{array}$ & 1 & $\begin{array}{l}\text { feeding, } \\
\text { changing } \\
\text { diaper, } \\
\text { giving bath, } \\
\text { comforting, } \\
\text { putting to } \\
\text { sleep, } \\
\text { playing }\end{array}$ & 6 & $\begin{array}{l}\text { feeding, } \\
\text { changing } \\
\text { diaper, } \\
\text { giving bath, } \\
\text { comforting, } \\
\text { putting to } \\
\text { sleep, } \\
\text { playing }\end{array}$ & $30 \mathrm{~s}$ & Unemployed & 5 & 59 \\
\hline M & $30 \mathrm{~s}$ & $5: 00$ & 19:00 & $\begin{array}{l}2 \text { days/ } \\
\text { week }\end{array}$ & 1 & $\begin{array}{l}\text { cooking, } \\
\text { laundry, } \\
\text { cleaning, } \\
\text { taking out } \\
\text { trash, } \\
\text { shopping }\end{array}$ & 5 & $\begin{array}{l}\text { cooking, } \\
\text { laundry, } \\
\text { cleaning, } \\
\text { taking } \\
\text { out } \\
\text { trash, } \\
\text { shopping }\end{array}$ & 2 & $\begin{array}{l}\text { feeding, } \\
\text { changing } \\
\text { diaper, } \\
\text { giving bath, } \\
\text { comforting, } \\
\text { playing }\end{array}$ & 5 & $\begin{array}{l}\text { feeding, } \\
\text { changing } \\
\text { diaper, } \\
\text { giving bath, } \\
\text { comforting, } \\
\text { putting to } \\
\text { sleep, } \\
\text { playing }\end{array}$ & $30 \mathrm{~s}$ & Unemployed & 6 & 73 \\
\hline $\mathrm{N}$ & $30 \mathrm{~s}$ & $7: 00$ & $18: 00$ & $\begin{array}{c}2 \text { days/ } \\
\text { week }\end{array}$ & 0.5 & $\begin{array}{l}\text { taking out } \\
\text { trash, } \\
\text { doing } \\
\text { dishes, } \\
\text { shopping }\end{array}$ & 0.5 & $\begin{array}{l}\text { cooking, } \\
\text { shopping }\end{array}$ & 1.5 & $\begin{array}{l}\text { feeding, } \\
\text { changing } \\
\text { diaper, } \\
\text { giving bath, } \\
\text { comforting, } \\
\text { playing }\end{array}$ & 5 & $\begin{array}{l}\text { feeding, } \\
\text { changing } \\
\text { diaper, } \\
\text { giving bath, } \\
\text { comforting, } \\
\text { putting to } \\
\text { sleep, } \\
\text { playing }\end{array}$ & $30 \mathrm{~s}$ & Part-time & 4 & 63 \\
\hline $\mathrm{O}$ & $30 \mathrm{~s}$ & $7: 00$ & 19:00 & $\begin{array}{l}1 \text { day/ } \\
\text { week }\end{array}$ & 1 & $\begin{array}{l}\text { doing } \\
\text { dishes }\end{array}$ & 1 & $\begin{array}{l}\text { cleaning, } \\
\text { doing } \\
\text { dishes }\end{array}$ & 4 & $\begin{array}{l}\text { changing } \\
\text { diaper, } \\
\text { giving bath, } \\
\text { comforting }\end{array}$ & 1 & $\begin{array}{l}\text { changing } \\
\text { diaper, } \\
\text { giving bath, } \\
\text { comforting }\end{array}$ & $20 \mathrm{~s}$ & $\begin{array}{l}\text { On } \\
\text { maternity } \\
\text { leave }\end{array}$ & 5 & 69 \\
\hline
\end{tabular}


The background of the subjects is shown in Table 1 . While initially 17 subjects gave verbal consent, we were able to contact and obtain the consent of only 15 subjects 3 months later. The average age of the child of each subject was 5.5 months. The age distribution was 3 subjects in 20 years, and 12 in 30 years. The average time spent doing housework was 80 minutes. The average time spent caring for the child was 210 minutes.

2) Difficulties associated with paternal roles (Table 2)

Table 2 shows the difficulties associated with paternal roles. We analyzed the difficulties faced by fathers with a focus on the period from pregnancy through early infancy. 268 recording units were extracted from the transcripts. Categorizing the content based on similarity yielded five primary categories: $<$ Difficulties during early engagement with child $>$, $<$ Difficulties related to child crying $>$, $<$ Difficulties arising after getting through the early engagement stage $>,<$ Difficulties related to support of partner $>$, and $<$ Mental burdens associated with childrearing $>$. Within these are 11 secondary categories and 33 tertiary categories. Within this document, the following formatting is used to differentiate the levels of categorization: <primary categories>, \{secondary categories\}, [tertiary categories], and "subject narration".

a) <Difficulties during early engagement with child $>$

This category concerns the difficulties father faced during the early stages of connecting and involving themselves with their child. It consists of two secondary categories: $\{$ Fear/confusion concerning first-time childcare skills $\}$ and $\{$ Need time for the reality of being a father to sink in\}.

\{Fear/confusion concerning first-time childcare skills\} breaks down further into three tertiary categories, [Confusion with first-time childcare skills], [Feelings of fear due to lack of experience], and [Anxiety from not knowing how to interact with child]. These are made up of phrases concerning difficulties with basic childcare skills coming from a lack of experience in dealing with their first child, such as "I was so scared giving it a bath. I had my wife stay next to me." and "I was scared. I didn't know how to hold it. The neck was so scary. I didn't even know how hard I should pat its back".

\{Need time for the reality of being a father to sink in $\}$ breaks down further into two tertiary categories: [Reality of being a father hasn't sunk in by birth] and [Reality hasn't sunk in for several days after birth]. These are made up of phrases concerning feelings of fear and difficulty developing a familial connection with the child before they came to terms with the reality that a child had entered their personal life, both during pregnancy and during/after childbirth, such as "I didn't understand my picture of a father. It didn't hit me until after (the baby) was born..." and "Personally, at first it felt weird, like this being had come home with us. Almost like getting a new pet."

b) <Difficulties related to child crying >

This category concerns the most distinct source of difficulty, the continual crying of the infant. It consists of two secondary categories: \{Distress from child crying and $\{$ Dealing with crying through trial and error\}. 
Table 2. Difficulties associated with paternal roles.

\begin{tabular}{|c|c|c|c|c|}
\hline $\begin{array}{l}\text { Primary } \\
\text { Category }\end{array}$ & Secondary Category & Tertiary Category & & s (\%) \\
\hline \multirow{5}{*}{$\begin{array}{l}\text { Difficulties } \\
\text { during early } \\
\text { engagement } \\
\text { with child }\end{array}$} & & Confusion with first-time childcare skills & 26 & \multirow{5}{*}{$(21.3)$} \\
\hline & $\begin{array}{l}\text { Fear/confusion concerning first-time } \\
\text { childcare skills }\end{array}$ & Feelings of fear due to lack of experience & 14 & \\
\hline & & Anxiety from not knowing how to interact with child & 7 & \\
\hline & \multirow{2}{*}{$\begin{array}{l}\text { Need time for the reality of being a } \\
\text { father to sink in }\end{array}$} & Reality of being a father hasn't sunk in by birth & 7 & \\
\hline & & Reality hasn't sunk in for several days after birth & 3 & \\
\hline \multirow{8}{*}{$\begin{array}{l}\text { Difficulties } \\
\text { related to } \\
\text { child crying }\end{array}$} & \multirow{7}{*}{ Distress from child crying } & Confusion from child crying & 13 & \multirow{8}{*}{$(20.9)$} \\
\hline & & Agitation from child crying & 6 & \\
\hline & & Sense of powerlessness from child crying & 4 & \\
\hline & & Sense of uncertainty from child crying & 6 & \\
\hline & & Frustration from child crying & 7 & \\
\hline & & Sense of resentment from child crying & 3 & \\
\hline & & Dealing with crying through trial and error & 11 & \\
\hline & Dealing with crying through trial and error & $\begin{array}{l}\text { Dealing with crying as a couple through trial and } \\
\text { error }\end{array}$ & 6 & \\
\hline \multirow{6}{*}{$\begin{array}{l}\text { Difficulties } \\
\text { arising after } \\
\text { getting through } \\
\text { the early } \\
\text { engagement } \\
\text { stage }\end{array}$} & $\begin{array}{l}\text { Sense of distance until the infant's response to } \\
\text { father can be understood }\end{array}$ & Deepening of affection from understanding reactions & 12 & \multirow{6}{*}{$(10.1)$} \\
\hline & \multirow{2}{*}{ Concern about illness, injury, and accidents } & Concern about accidents and injuries & 4 & \\
\hline & & Confusion about illness & 2 & \\
\hline & \multirow{3}{*}{$\begin{array}{l}\text { Concern associated with growth/ } \\
\text { development }\end{array}$} & $\begin{array}{l}\text { Realizing the difficulty of taking care of child as it } \\
\text { grows }\end{array}$ & 2 & \\
\hline & & Concern about future injuries, accidents, or disease & 5 & \\
\hline & & Anxiety about growth/development & 2 & \\
\hline \multirow{7}{*}{$\begin{array}{l}\text { Difficulties } \\
\text { related to } \\
\text { support of } \\
\text { partner }\end{array}$} & \multirow{5}{*}{$\begin{array}{l}\text { Consideration toward partner during } \\
\text { pregnancy and after childbirth }\end{array}$} & Supporting partner before pregnancy & 2 & \multirow{7}{*}{ (29.9) } \\
\hline & & Consideration toward partner during pregnancy & 18 & \\
\hline & & Consideration toward partner after childbirth & 18 & \\
\hline & & Thoughtful communication & 8 & \\
\hline & & Concern for partner's mental health & 11 & \\
\hline & \multirow{2}{*}{ Marital difficulties } & Pressure from partner & 13 & \\
\hline & & Conflict with partner & 10 & \\
\hline \multirow{7}{*}{$\begin{array}{l}\text { Mental burdens } \\
\text { associated with } \\
\text { childrearing }\end{array}$} & \multirow{2}{*}{ Exhaustion/Sleep deprivation after birth } & Exhaustion after child is born & 8 & \multirow{7}{*}{$(17.9)$} \\
\hline & & Sleep deprivation after child is born & 4 & \\
\hline & \multirow{5}{*}{ Various stressors } & Lack of personal time & 5 & \\
\hline & & Stress from lifestyle & 11 & \\
\hline & & Need to release stress & 13 & \\
\hline & & Unease or constraint related to partner's family & 4 & \\
\hline & & Pressure from workplace & 3 & \\
\hline
\end{tabular}


\{Distress from child crying\} breaks down further into tertiary categories concerning fathers' experiences falling into a state of crisis from being unable to stop the infant from crying no matter how desperate the attempt and the many emotions they feel in these situations: [Confusion from child crying], [Agitation from child crying], [Sense of powerlessness from child crying], [Sense of uncertainty from child crying], [Frustration from child crying], and [Sense of resentment from child crying]. These are made up of phrases such as "(The baby) cries and gets cranky even if I hold it. It's not hungry, its diaper is empty. No matter how much I cradle and rock it, it just won't stop. Times like that are mentally (hard).", "It drives me insane. I can't stand when it cries.", “(The baby) just won't stop crying even if I hold it. It's pretty frustrating.", "I can't help but sigh when it keeps crying. When it cries hard it makes my ears hurt. Even when I'm patting its back, sometimes I just want to say, 'I've had it' and yell at it to shut up'."

\{Dealing with crying through trial and error\} breaks down further into categories concerning the difficulties fathers and their partners face in trying various methods to stop the crying despite not knowing how to respond. These are made up of phrases such as "When (the baby) is crying hard, I freak out, not knowing what to do. I'll try for 15 or 20 minutes, holding it, walking around, standing, sitting, but it just keeps crying.", "It was the middle of the night, and my wife was tired. I was so desperate to give (the baby) some milk. We were both exhausted."

c) $<$ Difficulties arising after getting through the early engagement stage $>$

This category concerns the difficulties fathers face after having become accustomed to their child and interacting with it proactively. It consists of three subcategories: \{Sense of distance until the infant's responses to father can be understood\}, \{Concern about illness, injury, and accidents\}, and \{Concerns associated with growth/development\}.

The category \{Sense of distance until the infant's responses to father can be understood\} consists of the subcategory [Deepening of affection from understanding reactions]. Fathers spoke of becoming more comfortable with their child's responses as they developed, but also of the anxiety they felt when unsure of a response, using phrases such as: "(The baby) laughs when I play with it, and that's reassuring. At first it just smiled a little, but now it laughs out loud. It's cute.", "(The baby) loves it when I sing a song and move its hand along with the rhythm. It's so cute when it coos. At first, it always ignored me when I came home."

The category \{Concern about illness, injury, and accidents\} consists of subcategories [Concern about accidents and injuries] and [Confusion about illness]. Fathers spoke of being unable to respond to unexpected changes in their child's condition that they hadn't yet experienced.

The category \{Concern associated with growth/development consists of the subcategories [Realizing the difficulty of taking care of child as it grows], [Concern about future injuries, accidents, or disease], and [Anxiety about growth/ development]. Subjects described their uncertainty about their child's develop- 
ment, potential accidents that could occur as it grows, and coming to realize how difficult raising a child will be.

d) $<$ Difficulties related to support of partner $>$

This category concerns one of the paternal roles, supporting the partner. It consists of two subcategories, \{Consideration toward partner during pregnancy and after childbirth\} and \{Marital difficulties\}.

\{Consideration toward partner during pregnancy and after childbirth\} includes the subcategories [Supporting partner before pregnancy], [Consideration toward partner after childbirth], and [Consideration toward partner after childbirth], which concern the difficulties fathers face while providing instrumental support to their partner before, during, and after the pregnancy. Some phrases include: "While she was pregnant, I would vacuum and other things, but she didn't like when I did it in front of her, so I waited until she wasn't home." and "I watch her expression, like 'Oh, something's bothering her.' I do the dishes, wash and hang the laundry, and give the baby a bath." This category also included the subcategories [Thoughtful communication] and [Concern for partner's mental health], which consist of subjects' difficulties related to providing emotional support to avoid placing a mental burden on their partner that would negatively affect the child. Subjects used phrases such as "She was really unstable when she was pregnant. Sometimes mean. But I would just brush it off."

The category \{Marital difficulties\} consists of the subcategories [Pressure from partner] and [Conflict with partner], which concern the difficulties based on friction and inequality in subjects' relationship with their partner. Subjects used phrases such as "She gets mad sometimes. Really biting my head off." and "If I messed up while I was wiping the baby and it got everywhere, she would get mad and say, 'What are you doing?!' I really didn't like that atmosphere."

e) $<$ Mental burden associated with life as a parent $>$

This category concerns burdens the subjects met with following childbirth while performing their parental responsibilities. It consists of the subcategories \{Exhaustion/Sleep deprivation after birth\} and \{Various stressors\}.

\{Exhaustion/Sleep deprivation after birth\} consists of the [Exhaustion after child is born] and [Sleep deprivation after child is born] subcategories, which pertain to difficulties associated with the workplace and life as a parent following the birth of the child, based on phrases such as "I dozed off at the convenience store on the way home from work. I was exhausted from work and hadn't slept the night before. I didn't think I would get any sleep that night either."

\{Various stressors\} consists of the [Lack of personal time], [Stress from lifestyle], [Need to release stress], [Stress from lifestyle], [Need to release stress], [Unease or constraint related to partner's family], and [Pressure from workplace] subcategories, which pertain to mental stress associated with the changes in lifestyle after the birth of the child. Phrases include: "Sometimes I just want some alone time.", "It's actually more tiring to be at home. I prefer to be at work. I have to adjust myself to what the baby needs, do the shopping, do housework... I have to control my emotions at work, but at home I can't. My wife is mad at 
me, and the baby is crying...".

3) Desired support for maintaining mental health (Table 3)

We analyzed what types of support subjects needed pre- and post-birth. The results of this analysis are shown in Table 3. 92 recording units were extracted and categorized based on the semantic content. The table includes the primary categories <Support with childcare knowledge/acquiring skills $>$, <Support in building a positive marital relationship $>$, and $<$ Support in maintaining own mental health $>, 8$ secondary categories, and 17 tertiary categories. These are formatted as follows to aid in distinguishing the levels: $<$ Primary categories $>$, \{Secondary categories\}, [Tertiary categories].

$<$ Support with childcare knowledge/acquiring skills> consists of the subcategories \{Childcare knowledge/acquiring skills\} and \{Dealing with crying\}, which in turn consist of subcategories such as [Help with basic childcare skills] and [Information/management strategies for growth/development].

$<$ Support in building a positive marital relationship $>$ consists of four secondary categories: $\{$ Empathizing with wife\}, consisting of [Pregnancy-related support] and [Support regarding childbirth with father present]; \{Help with providing

Table 3. Desired support for maintaining mental health.

\begin{tabular}{|c|c|c|c|c|}
\hline Primary Category & Secondary Category & Tertiary Subcategory & & $(\%)$ \\
\hline \multirow{3}{*}{$\begin{array}{l}\text { Support with childcare } \\
\text { knowledge/ } \\
\text { acquiring skills }\end{array}$} & & Help with basic childcare skills & 5 & \multirow{3}{*}{$(12.0)$} \\
\hline & $\begin{array}{l}\text { Childcare knowledge/ } \\
\text { acquiring skills }\end{array}$ & $\begin{array}{l}\text { Information/management strategies for } \\
\text { growth/development }\end{array}$ & 2 & \\
\hline & Dealing with crying & Causes of and coping strategies for crying & 4 & \\
\hline \multirow{7}{*}{$\begin{array}{l}\text { Support in building a } \\
\text { positive marital } \\
\text { relationship }\end{array}$} & & Pregnancy-related support & 1 & \multirow{7}{*}{$(54.3)$} \\
\hline & & Support regarding childbirth with father present & 2 & \\
\hline & & Housework-related support & 9 & \\
\hline & $\begin{array}{l}\text { Help with providing maternal } \\
\text { support }\end{array}$ & $\begin{array}{l}\text { Help with changes in mother's state of mind and } \\
\text { providing support for mother }\end{array}$ & 7 & \\
\hline & $\begin{array}{l}\text { Help with childrearing support } \\
\text { environment }\end{array}$ & Help with community support & 4 & \\
\hline & \multirow{2}{*}{ Maintaining marital relationship } & Help regarding interspousal communication & 6 & \\
\hline & & Help with maintaining interspousal power balance & 4 & \\
\hline \multirow{5}{*}{$\begin{array}{l}\text { Support in maintaining } \\
\text { own mental health }\end{array}$} & & Information to help imagine life after childbirth & 5 & \multirow{5}{*}{$(33.7)$} \\
\hline & $\begin{array}{l}\text { Information concerning paternal } \\
\text { mental health }\end{array}$ & $\begin{array}{l}\text { Information regarding maintaining paternal } \\
\text { mental health }\end{array}$ & 4 & \\
\hline & & $\begin{array}{l}\text { Information to help mother understand } \\
\text { father's feelings }\end{array}$ & 9 & \\
\hline & \multirow{2}{*}{ Support related to stress relief } & $\begin{array}{l}\text { Help creating an environment in which } \\
\text { fathers can discuss their needs }\end{array}$ & 4 & \\
\hline & & Anger-management-related support & 3 & \\
\hline
\end{tabular}


maternal support\}, consisting of [Housework-related support] and [Help with changes in mother's state of mind and providing support for mother]; \{Help with childrearing support environment\}, consisting of [Help with familial support] and [Help with community support]; and \{Maintaining marital relationship\}, consisting of [Help regarding interspousal communication] and [Help with maintaining interspousal power balance].

$<$ Support in maintaining own mental health $>$ consists of two secondary categories: \{Information concerning paternal mental health\}, consisting of [Information to help imagine life after childbirth], [Information regarding maintaining paternal mental health], and [Information to help mother understand father's feelings]; and \{Support related to stress relief\}, consisting of [Help creating an environment in which fathers can discuss their needs], [Help with securing personal time], and [Anger-management-related support].

\section{Discussion}

\section{1) Subject Characteristics}

The subjects' ages were average when compared to the average age of fathers at the time of birth of their first child, 32.8 years [22]. The average time spent doing housework per week was 1.4 hours, and the average time spent caring for the child was 3.5 hours per week. These were significantly longer than the results reported in the 2016 Survey on Time Use and Leisure Activities ( 0.17 hours/week for housework and 0.49 hours/week for childcare) [5].

2) Difficulties associated with paternal roles and support for the maintenance of mental health

It has become evident that hardship arises in new fathers with infants due to the mental burden caused by the two factors of paternal roles (engagement with child and supporting spouse). Subjects spoke on their difficulties and the support they need to maintain their mental health pre- and post-childbirth. We will examine fathers' educational support needs in terms of both the difficulties they have and the support they are looking for.

a) Difficulties and educational support needs related to engagement with child

The difficulties related to fathers' engagement with the child are made up of fear and confusion about childcare skills and the time necessary the sense of fatherhood to sink in. Following these, the difficulties that arise after early engagement stage include the sense of distance until fathers understand their child's reactions to them; concerns about illness, injury, and accidents; and concern over growth and development.

After bringing the baby into their home, fathers gradually got used to it, and through contact with it began to understand their child's reactions and how it became more aware of the father. It is evident that a loving relationship between father and child is formed through the difficulties they experience. It is also clear that this close relationship brings about new difficulties based in worries about illness and accidents. 
For both mothers and fathers, parenthood begins to develop during pregnancy [23] [24] [25]. The realization of paternal role behaviors continues from gestation through the perinatal period [26], and both men and women develop as parents [27]. Awareness as a father is a vital component in developing a sense of parenthood. It does not spontaneously occur during pregnancy or immediately after birth; rather, it gradually builds through interaction with the child while contending with the fear and uncertainty surrounding parenting skills. As a father raises his child, he develops an intense emotional interest in it, called "engrossment" and begins to feel a variety of positive emotions. This engrossment is said to bring increased feelings of self-worth and cause fathers to recognize themselves and their role as a father [28].

Thus, it can be said that the difficulties faced in the early engagement stage are a necessary part of the process of building an affinity with the child as he performs his parental roles while contending with feelings of fear and distance associated with it.

The educational needs of fathers in terms of basic parenting skill support were extracted from the interviews. Taking practice parenting courses, which are common in current times, during pregnancy is important for increasing the sense of parenthood, but the subjects of this study were unable to attend prenatal courses due to the COVID-19 pandemic. Many fathers used online childcare videos as a reference. In practice, all subjects acquired childcare skills through guidance from the mothers postpartum, which the fathers were comfortable with. The building of a new relationship with the mother through parenting can be thought of as a necessary part of adapting from life as a couple to life with a child.

Determinants for paternal participation in childcare include motivation, childcare skills, support from significant others (wife, family, friends and colleagues) and four institutional factors [29]. One of those factors is support from the mother. It is necessary to assist the mother so she is able to support the father in childcare tasks, as well as to communicate that there is no absolute right or wrong method of childcare, so as not to interrupt the engrossment process.

The worst difficulty fathers had when interacting with their child was the constant crying, which stirred up tangles of negative emotions such as confusion, agitation, powerlessness, uncertainty, resentment, and frustration. Strong ambivalent or negative responses such as getting annoyed at the noise despite thinking the infant is cute or getting irritated that the infant will not stop crying indicate a high level of stress is involved [30]. It was suggested that fathers fell into a state of crisis in the face of non-stop crying. At the same time, however, fathers continued to face this difficulty and build a relationship with the infant. They expressed that they were "saved" by their child's smile and happy response to them. It is clear that the father-infant relationship deepens while fathers are at the mercy of these conflicting attitudes toward the child.

When the infant cried, the parents attempted to deal with the situation through trial and error even though they did not know what to do. When noth- 
ing worked, both fathers and mothers took showed evasive behavior and left the other spouse to take care of the infant. While mothers reach a state of crisis when they realize their baby will not stop crying no matter what is done, it is reported that the availability of support from the husband or others has a major effect on whether or not the mother succumbs to the stress [31]. Cooperation between spouses in dealing with crying is important.

From the interview data, we extracted a need for educational support on how to deal with a crying infant. This study clearly illustrates the mental state of fathers who are faced with constant crying, from feelings of confusion to irritation. It is presumed that the risk of mistreatment is increased in cases where the crying does not stop, and the father reaches a state of crisis [32] [33]. It is important to recognize the circumstances of facing constant crying beforehand, and to provide support so one parent is not dealing with it alone and both parents can manage it cooperatively.

b) Difficulties related to supporting wife and educational support needs

The difficulties fathers experience related to supporting their wives consisted of special consideration for the wife both during pregnancy and postpartum, as well as relationship difficulties with the wife. Fathers supported their wives before, during, and after pregnancy by helping with childcare/housework and providing emotional support through considerate communication. It is clear that subjects felt some difficulty in relation to this support, and particularly that subjects felt pressure from their wives and occasionally had confrontations with them.

It is said that during the transition to being parents, couples experience fluctuations in their level of closeness and grow apart [34]. 50\% of couples experience a worsening of their relationship [35], with closeness continuing to fall two months [36], four months [37], six months [38], eighteen months [39], and two or three years [40] following childbirth.

It has been shown that changes take place in five domains during the transition to parenthood: self-identity, marital roles and relationship, intergenerational roles and relationships, roles and relationships outside the home, and roles and relationships as a new parent [41]. While women show a $70 \%$ increase in their sense of motherhood from 6 - 8 months postpartum, men show only an increase of approximately $30 \%$ in their sense of fatherhood [41]. The majority of fathers are still seen as workers in society through this period [41].

Domestic factors contribute to the decline in closeness. From the perspective of the wife there is a lack of parenting [42] [43] [44] and personal [44] support from the husband, and this continues to have an influence 4 months [45], six months [38], one year [46], and two years [47] following the birth. On the other hand, husbands were more nervous during pregnancy than their wives [48], and working hours and irritation from the wife were a major factor. Over a three-year period staring during pregnancy, husbands had high patience scores; there is a tendency for husbands to try to be patient and attune themselves to their wives' 
moods during daily life [40].

Given the differences in the rate of change in identity between spouses, wives get angry when paternal roles-especially those related to the support of the wife-are not performed according to their expectations. This leads the husband to feel pressured and may sometimes lead to confrontation.

From the interview data we extracted a need for educational support dealing with building a positive marital relationship. Closeness increases based on the husband's participation in parenting and housework [36] [49] [50] as well interspousal communication [51]. There is a need for support related to thoughtful communication between spouses, and methods of participating in housework and childcare as much as one can in order to satisfy the expectations of the mother, as well as enlisting the help of grandparents, who can support the parents with housework and childcare to the mother's satisfaction, and maintaining an environment of community support.

c) Physical and mental burdens on fathers and their educational support needs

The physical and mental burdens on fathers include exhaustion/sleep deprivation and various stresses following the birth. The pressures of work and home life are reflected concretely in exhaustion and sleep deprivation. It is clear that fathers feel a lack of time and a need to release the many stresses of their lives. These difficulties are multi-layered, originating from hardships associated with two paternal roles-being involved with the infant and supporting the wife.

Because the idea that fathers are workers remains after the birth of the child [41], they diligently work during the daytime and attend to the child and wife while at home. Late-night childcare tasks such as feeding and dealing with crying take their toll and the physical and mental burden grows heavier.

Positive aspects of the performance of paternal roles include feelings of love, responsibility, presence of mind, broadened perspectives, and outlook on the past and future, while a negative aspect is a loss of personal freedom [52]. Men have an insubstantial image of the negative aspects during pregnancy [52]. They respond negatively after the birth, and without confidence in their ability to raise the child, they feel more strongly the negative aspects. In our competitive and controlled society, these fathers are saddled with the pressures of the workplace. Life after the birth is different than what they pictured during the pregnancy and the combined burden of work and home life causes them to see their paternal roles in a negative light, causing additional stress. If fathers are unable to cope with this stress, it will have a detrimental effect on their mental health.

We extracted from the interview data a need for support for fathers to maintain their mental health. This support would help fathers picture the stressful changes in life after the birth of a child before they occur, and provide information pertaining to paternal mental health. Specifically, it is important to also provide mothers with information on mental health and the difficulties associated with performing paternal roles in order to deepen their understanding of fathers' situations. Fathers have need of an environment in which they can con- 
sult others, as well as personal time. Stress relief is necessary to prevent detrimental effects on mental health.

Kawahara \& Sadahisa [53] report that the negative aspects of paternal roles are more strongly felt if the father does not speak about his child, though fathers find it easy to speak directly with longtime friends and coworkers. The subjects of our study also reported that they solved issues by speaking with colleagues who had experience raising children, which helped to alleviate stress. However, making friends with other fathers over the subject of raising a child is difficult in reality. While it is important for fathers to secure an environment in which one can consult with others, they must first make sure that their situation allows them to speak their mind in their circle of experienced friends and colleagues.

Many subjects in this study spoke of the great mental burden associated with performing paternal roles and forcing themselves to endure for the sake of the forming/maintaining their relationship with their wife and child. As it can be expected that further stress may lead to feeling of anger, anger management support is important as one form of mental health management available to fathers.

In recent years, there has been a tendency for paternal support to be actively practiced in keeping with fathers' parenting recommendations. However, the results of a nationwide survey of support in promoting the acquisition of paternal roles conducted by midwives, the majority of support is for mothers, consisting of labor support, nursing support, communication support, etc. [54]. As a result of surveying the subjects, this study has found two factors of paternal roles (childrearing and maternal support) and that the burdens associated with these roles are a cause of hardship. These are new findings.

The task moving forward is to investigate supplementing and revising current support practices to provide better educational support to fathers. Past group meetings saw attendance rates of not more than 10\% [55] and the widespread effects of the COVID-19 pandemic across the country made it difficult for fathers to attend. Further consideration into methods of instruction is needed.

3) Limitations

This research is the result of a qualitative study based on the interviews of 15 new fathers. Because subjects were the spouses of mothers who gave birth at one medical institution, there is a risk of sampling bias reflecting the institution's characteristics, as well as limitations to the generalization of the results. In the future, results should be carefully verified, having interviews conducted at other institutions, in other regions, and with a larger sample size.

The development of a practical, general guidebook usable by nursing staff preand postpartum is a task for the future. We will create an educational support guidebook for nursing staff based on the needs of new fathers found in this research with the aim of maintaining paternal mental health.

\section{Conclusions}

1) Five categories of difficulties associated with paternal roles were extracted 
from the data, with a focus on the period from pregnancy through early infancy: $<$ Difficulties during early engagement with child $>$, $<$ Difficulties related to child crying $>,<$ Difficulties arising after getting through the early engagement stage $>$, $<$ Difficulties related to support of partner $>$, and $<$ Mental burdens associated with childrearing $>$. The stress experienced by fathers from the birth of their child through early infancy, originating in two elements of fatherhood (engagement with the child and spousal support), placed a great mental burden upon them in a multi-layered structure.

2) Three categories of support for the purpose of maintaining paternal mental health pre- and postpartum were extracted from the data. These needs were based on the hardships they experienced in raising their child.

3) There is a disparity between fathers' educational support needs and current paternal support practices. The supplementation and revision of existing paternal support practices is an issue that merits further consideration. Furthermore, the existing group interview method suffers from low attendance, and attending has become increasingly difficult due to the COVID-19 pandemic spreading throughout Japan. It is necessary to consider alternative methods of educational support moving forward.

\section{Acknowledgements}

We would like to express our gratitude to the fathers and maternity ward staff for their cooperation with this study.

\section{Conflicts of Interest}

The authors declare no conflicts of interest regarding the publication of this paper.

\section{References}

[1] Japanese Ministry of Health, Labour and Welfare (2020) Vital Statistics Monthly Report Annual Survey (Approximate Number) Overview 2019. https://www.mhlw.go.jp/toukei/saikin/hw/jinkou/geppo/nengai19/dl/kekka.pdf

[2] Japanese Cabinet Office (2020) Overview of the Situation of Declining Birthrates and Measures to Cope with Declining Birthrates in 2019 Fiscal Year. https://www8.cao.go.jp/shoushi/shoushika/whitepaper/measures/w-2020/r02pdfgai yoh/pdf/02gaiyoh.pdf

[3] Japanese Cabinet Office (2016) Japan 100 Million Total Active Plan. https://www.kantei.go.jp/jp/singi/ichiokusoukatsuyaku/pdf/plan1.pdf

[4] Japanese Cabinet Office (2020) Progress in Measures to Reduce the Birthrate. https://www8.cao.go.jp/shoushi/shoushika/data/pdf/torikumi.pdf

[5] Japanese Ministry of Internal Affairs and Communications (2017) Summary of the Results of the 2016 Basic Survey on Social Life and Living Time. http://www.stat.go.jp/data/shakai/2016/pdf/youyaku2.pdf

[6] Japanese Ministry of Health, Labour and Welfare (2020) Summary of Survey Results "2019 Basic Survey on Gender Equality in Employment Management”.

https://www.mhlw.go.jp/toukei/list/dl/71-r01/07.pdf 
[7] Japanese Cabinet Office-Gender Equality Bureau (2020) The Fifth Basic Plan for Gender Equality.

https://www.gender.go.jp/about_danjo/basic_plans/5th/pdf/5th_gaiyo.pdf

[8] Sasaki, H., Goto, A., Watanabe, H., Yamazaki, S., Kawai, T. and Yasumura, S. (2010) Paternal Confidence in Child Rearing during the First Year of Parenting among Japanese Fathers in a Local City: Part 1: Frequency of Lacking Confidence and Its Association with Other Parenting Indicators. The Journal of Child Health, 69, 790-795.

[9] Ando, A., Hiraoka, Y., Takeshima, H., Syouji, J., Kawai, N., Nakamura, K. and Kuwahara, K. (2010) A Fundamental Study on Child-Rearing Anxiety of Fathers (4) Analyzing the Parents' Attribution and the Factors Related to the Feeling of Difficulty with Child-Rearing. Reports of studies of Japan Child and Family Research Institute, 47, 303-312.

[10] Feinberg, M.E. and Kan, M.L. (2008) Establishing Family Foundations: Intervention Effects on Coparenting, Parent/Infant Well-Being, and Parent-Child Relations. Journal of Family Psychology, 22, 253-263. https://doi.org/10.1037/0893-3200.22.2.253

[11] Enya, Y. and Kabeyama, K. (2018) Depression Status of Couples from Birth until 1 Month Postpartum. Journal of Japanese Society of Psychosomatic Obstetrics and Gynecology, 22, 299-306.

[12] Tokumitsu, K., Sugawa, N., Maruo, K., Suzuki, T., Yasui-Fukuori, N. and Shimodo, K. (2020) Prevalence of Perinatal Depression among Japanese Men: A Meta-Analysis. Annals of General Psychiatry, 19, 1-18. https://doi.org/10.1186/s12991-020-00316-0

[13] Paulson, J.F. and Bazemore, S.D. (2010) Prenatal and Postpartum Depression in Fathers and Its Association with Maternal Depression: A Meta-Analysis. JAMA, 303, 1961-1969. https://doi.org/10.1001/jama.2010.605

[14] Davis, R.N., Davis, M.M., Freed, G.L. and Clark, S.J. (2011) Father's Depression Related to Positive and Negative Parenting Behaviors with 1-Year-Old Children. Pediatrics, 127, 612-617. https://doi.org/10.1542/peds.2010-1779

[15] Japanese Cabinet Office Gender Equality Bureau (2020) The Fifth Basic Plan for Gender Equality-Part 2 Policy-Field 3: Promotion of Gender Equality in Local Communities. https://www.gender.go.jp/about_danjo/basic_plans/5th/pdf/2-03.pdf.

[16] Higashio, K. and Sasaki, A. (2020) A Literature Review on Education and Support for Fathers to Adapt to Paternal Roles during Infancy. Women's Health Society Journal of Japan, 19, 45-55.

[17] Takehara, K. (2019) Two-Week and One-Month Postpartum Checkups Assessment and Care of Mother and Child: Don't Overlook the Signs That Concern You, Such as Mental Health, Breastfeeding, and Newborn Development. Topics 2 Postpartum Depression in Fathers. Perinatal Care, 38, 985-988.

[18] Funahashi, K (2005) Gender in Family Policies and Child Care Strategies-Comparing the Experiences of Japan, France and Sweden. Japanese Journal of Family Sociology, 16, 23-35. https://doi.org/10.4234/jjoffamilysociology.16.2_23

[19] Yamato, R., Onode, S. and Kiwaki, T. (2008) Men's Child Care and Women's Child Care: An Approach from Family Sociology. Showado, Kyoto 165.

[20] Okada, A., Nishimura, K., Murata, M. and Matsui, H. (2018) Conceptual Analysis of Father's Role. Japanese Journal of Maternal Health, 59, 398-405.

[21] Berelson, B. (1957) Social Psychology Course 7. The Masses and Mass Communication, Misuzu Shobo, Ltd., Tokyo.

[22] Japanese Ministry of Health, Labour and Welfare (2019) Vital Statistics, Average 
Age of Father by Year, by Birth Order (E-Stat). https://www.e-stat.go.jp/stat-search/file-ownload?\&statInfId=000031981523\&fileKi $\underline{\mathrm{nd}=1}$

[23] Onodera, A., Aoki, K. and Koyama, M. (1998) Becoming a Father: Psychological Processes. The Japanese Journal of Developmental Psychology, 9, 121-130.

[24] Sasaki, K., Ueda, A., Suzuki, Y., Maeda, T. and Katayama, R. (2004) Does Shaping Parental Consciousness Relate to Concern for the Unborn Child and Marital Relationship during Pregnancy? The Journal of the Yonago Medical Association, 55, 142-150.

[25] Ochiai, H., Sasano, K., Kitahama, M., Tsubomoto, T., Yoritsune, K. and Yamazaki, S. (2012) The Development of Parenthood of First-Time Parents-From Pregnancy to 1 Month Postpartum. Hokuriku Journal of Public Health, 39, 1-5.

[26] Kigoshi, I. and Tomari, Y. (2006) Process of Acquisition of Paternal Role by Husbands during Perinatal Period. Japanese Journal of Research in Family Nursing, 12, 32-38.

[27] Kashiwagi, K. and Wakamatsu, M. (1994) "Becoming a Parent" and Personality Development: A Lifespan Developmental View. The Japanese Journal of Developmental Psychology, 5, 72-83.

[28] Martin Greenberg, M.D. (1994) The Birth of a Father. Takeuchi, T. Translation, Medicus Shuppan, Publishers Co., Ltd., Suita.

[29] Lamb, M.E., Pleck, J.H., Charnov, E.L. and Levine, J.A. (1987) A Biosocial Perspective on Parental Behavior and Involvement. In: Sherrod, L.R., Altmann, J., Rossi, A.S., Eds., Parenting across the Life Span Biosocial Dimensions, Routledge, London, 130-135.

[30] Iwata, H., Mori, E. and Maehara, S. (1998) Father's Stress on the Adaptation to Fatherhood and Its Predictors. Journal of Japan Academy of Nursing Science, 18, 21-36. https://doi.org/10.5630/jans1981.18.3_21

[31] Okamoto, M. and Matsuoka M. (2003) Postpartum Crisis of Primiparous Mother Facing Persistent Crying of 1-2-Month-Old Babies. Journal of JSPOG, 8, 58-92.

[32] Fujiwara, T., Yamada, Y. and Morisaki, N. (2016) Self-Reported Prevalence and Risk Factors for Shaking and Smothering among Mothers of 4-Month-Old Infants in Japan. Journal of Epidemiology, 26, 4-13. https://doi.org/10.2188/jea.JE20140216

[33] Yamada, F. and Fujiwara, T. (2014) Prevalence of Self-Reported Shaking and Smothering and Their Associations with Co-Sleeping among 4-Months-Old Infants in Japan. International Journal of Environmental Research and Public Health, 11, 64856493. https://doi.org/10.3390/ijerph110606485

[34] Ito, Y. (2015) A Review of Research on Characteristics of Intimacy in Marital relationships. The Japanese Journal of Developmental Psychology, 26, 279-287.

[35] Belsky, J. and Kelly, J. (1995) Translation to Parenthood. Ajimine, K. Translation, Soshisha Publishing Co., Ltd., Tokyo.

[36] Sugi, Y. and Katori, Y. (2017) The Changing of a Couple's Relationships across the Transition to Parenthood and Its Effect Factors: Focusing on the Period between Late Pregnancy and Two Months Postpartum. Japanese Journal of Maternal Health, 58, 296-305.

[37] Nagata, M., Nakamichi, Y., Noguchi, Y. and Hirata, N. (2011) Childrearing Stress Coping Strategies in Mothers at 1 and 4 Months Postpartum: Positivity in Childrearing. Japanese Journal of Maternal Health, 51, 609-615.

[38] Wakita, M., Kojima, Y. and Irisawa, M. (2003) Effects of Pregnancy and Childbirth 
on Mothers' Psychology: Focusing on Support from Husbands. Japanese Journal of Maternal Health, 44, 244-249.

[39] Miyanaka, F. (2002) Longitudinal Study of Changes in the Mother's Consciousness after Childbirth and Related Paternal Factors: Comparison between Primiparas and Multiparas during 18 Months after Childbirth. Journal of JSPOG, 7, 95-107.

[40] Onodera, A. (2005) Marital Changes during the Transition to Parenthood. The Japanese Journal of Developmental Psychology, 16, 15-25.

[41] Cowan, A.P. and Cowan, P.C. (2007) When Partners Become Parents: The Big Life Change for Couples. Yamada, M. and Hirauchi, A. Translation, Keiso Shobo Co., Ltd., Tokyo.

[42] Sato, S. (2012) Study on the Relationship of Various Factors Comprising a Sense of Satisfaction from Child Rearing and Assessment of the Marital Relationship as Pertains to Primiparas-Focusing on the Nuclear Family during the Initial Stage of Childrearing. Journal of Japan Academy of Midwifery, 26, 222-231. https://doi.org/10.3418/jjam.26.222

[43] Belsky, J., Lang M. and Huston T.L. (1986) Sex Typing and Division of Labor as Determinants of Marital Change across the Transition to Parenthood. Journal of Personality and Social Psychology, 50, 517-522. https://doi.org/10.1037/0022-3514.50.3.517

[44] Nakajima, K., Hayakawa, Y. and Tokiwa, Y. (2016) Husband and Wife's Relationship during Pregnancy and after Childbirth: Correlation between: "Satisfaction with Marital Relationship" and "Wives' Satisfaction with Husbands' Supportiveness". Japanese Journal of Maternal Health, 57, 82-89.

[45] Biefle, S.N. and Mickelson, K.D. (2012) First-Time Parents' Expectation about the Division of Childcare and Play. Journal of Family Psychology, 26, 36-45. https://doi.org/10.1037/a0026608

[46] Ruble, D.N., Fleming, A.S., Hackel, L.S. and Stangor, C. (1988) Change in the Marital Relationship during the Transition to First Time Motherhood: Effects of Violated Expectations Concerning Division of Household Labor. Journal of Personality and Social Psychology, 55, 78-87. https://doi.org/10.1037/0022-3514.55.1.78

[47] Maruyama, T., Yoshida, Y. and Sugiyama, A. (2004) The State of Psychosocial of Women during Pregnancy and 2 Years after Birth-Marital Relationship, Child-Rearing and Psychosocial Health Two Years after Childbirth. Journal of Japanese Society of Psychosomatic Obstetrics and Gynecology, 9, 74-81.

[48] Onodera. A. (2003) Changes in Self-Concept in the Transition to Parenthood. The Japanese Journal of Developmental Psychology, 14, 180-190.

[49] Sakanashi, K., Katsukawa, Y., Mizuno, S. and Kato, C. (2015) Mental Health and Child-Rearing Activities of Mothers with 1-Month-Old Infants. Kanto Gakuin University Journal of Nursing, 2, 1-9.

[50] Tombeau, C.K., Jonas, W., Untermaehre, E., Dudin, A., Sxatmari, P., Gaudeau, H., Kennedy, J., Atkinson, L., Steine, M., Meaney, M. and Fleming, A. (2018) Maternal Perceptions of Paternal Investment Are Associated with Relationship Satisfaction and Breastfeeding Duration in Humans. Journal of Family Psychology, 32, 1025-1035. https://doi.org/10.1037/fam0000468

[51] Ahlborg, T., Dahlof, L.G. and Hallberg, L.R. (2005) Quality of Intimate and Sexual Relationship in First-Time Parents Six Months after Delivery. The Journal of Sex Research, 42, 167-174. https://doi.org/10.1080/00224490509552270

[52] Morishita, Y. (2006) The Effect of Becoming Fathers on Men's Development. The Japanese Journal of Developmental Psychology, 17, 82-192. 
[53] Kawahara, Y. and Sadahisa M. (2018) Consciousness Investigation of Fathers with Infants and Toddlers. The Journal of Human Development and Clinical Psychology, 24, 45-50.

[54] Isoyama, A. (2015) Duty of Midwives Support to Encourage the Father Role Acquisition and Its Related factors. Journal of Japan Academy of Midwifery, 29, 230-239. https://doi.org/10.3418/jjam.29.230

[55] Adachi, Y. (2020) A Survey of Efforts to Encourage Fathers to Participate in Parenting in the Setting of Municipal Prenatal Education. Setsunan University Nursing Research, 8, 55-62. 